free from the disease; and inspectors should be appointed to make daily visits to their respective districts, and report all cases of diarrhœea, etc. Supplies of medicine for the treatment of diarrhœea should be lodged with them, and made readily available to all applicants; and extra allowances in the shape of beef-tea, etc., should be given to those of the poorer classes who might be attacked with diarrhoea.

One important feature deserving of attention was that, during the prevalence of cholera, the cases requiring medical attendance for other forms of disease were very exceptional; it seemed as if this dire epidemic had swallowed up all the minor ills.

\section{ON ANÆSTHETICS.*}

BY THOMAS J ONES, M.D.,

Assistant-Physician to Victoria Hospital for Sick Children; formerly (for eleven years) Resident Medical Officer and Administrator of Anæsthetics to

$$
\text { St. George's Hospital. }
$$

2. Ether was first employed by me as an anæsthetic at St. George's Ilospital in the year 1861. Although it was administered on a large conical sponge (a very objectionable mode), I succeeded in almost all cases in producing the necessary degree of narcotism. It was given for several capital operations, and in cases requiring careful dissection, such as hernix, with complete success. In the following year I discontinued its use, however, in the operating theatre, on account of the inconvenience occasioned to those present by the smell of the vapour, and from the time it took in some cases to get the patient under its influence, which was a very serious objection when several had to be operated upon. I, however, continued its use in cases which were considered unsafe for chloroform, such as those in a state of collapse from severe accidents. The stimulating effects of ether in such cases were attended with remarkably good results : they were generally in a better state after than before the operation.

In a paper read on this subject before St. George's Hospital Medical Society in 1863 , I observed that the result of my experience to that time tended to show (I) that ether caused during the first stage more cerebral excitement than chloroform ; (2) that it produced a greater degree of anæsthesia before the consciousness was completely abolished; (3) that during the stage of very complete unconsciousness, the reflex function of the cerebro-spinal centres was not abolished to the same degree as under chloroform ; (4) that it stimulates instead of depressing the heart.

My subsequent experience confirms, in the main, these conclusions. Ether, unlike chloroform, would seem to exert no paralysing influence on the heart, carried to the extent necessary for the performance of operations. In the paper referred to, I related two cases in which ether was administered with good effect after chloroform had been tried and found to produce dangerous symptoms.

Dr. Snow states (On Ancesthetics, p. 362) that "ether is altogether incapable of causing sudden death by paralysis of the heart, which has caused the accidents which have happened during the administration of chloroform. I have not been able to kill an animal in that manner with ether even when I have made it boil, and administered the vapour almost pure. The heart has continued to beat after the natural breathing has ceased, even when the vapour has been exhibited without air. .

I hold it, therefore, to be almost impossible that a death from this agent can occur in the hands of a medical man who is applying it with ordinary intelligence and attention". When this passage is taken in connection with the fact that Dr. Snow records in the same work no fewer than fifty fatal cases from chloroform, it is very wonderful that he should still persevere in the use of chloroform, especially when we find he observes (op. cit.) that he "usually rendered adult patients insensible in four or five minutes, and children in two or three", and that he "never failed to make the patient insensible in any one instance in which ether was administered". Hence, ether being in his hands perfectly successful and manageable, I cannot help agreeing with the opinion advanced in the leading article in this JouRNAL of the 16 th instant, that he, like every one else, was considerably influenced in the choice of the anæsthetic which he used by the energetic and uncompromising advocacy of the discoverer of chloroform.

I think the disadvantages of ether over chloroform are really very few and trivial. I find that some prefer the flavour of ether-vapour to that of chloroform. Its pungency, when inhaled, only lasts a few seconds, and not at all in some cases, when properly administered. As to the question of cost, I also think this objection has been exaggerated. Methylated washed ether (which answers all purposes quite as well) can be bought for half-a-crown a pound. If the anæsthetic be given

* Continued from page 577 of last number. rapidly and unsparingly at the commencement, I do not think the dose for each patient should cost more than sixpence-so that, in fact, the cost is not much greater than that of chloroform.

With reference to the mode of administration, I think the plan recommenaed by our American friends is as good as any-namely, a thick towel, in half-dozen folds, arranged in the form of a cone. Care should be taken that its base should be large enough to take in the chin and the nose-its shape such as to fit the face accurately. A small sponge should be placed inside, which should be squeezed out of warm water after each case, to prevent its becoming so cold as to interfere with the evaporation of the ether. Mr. Warrington Haward's apparatus, described in this JOURNAL on the 2 nd inst., is also a convenient method of administering it, and answers very well. But I find that the oiled silk, which forms its external layer, is an objection, because it interferes with the conduction of heat from the hand into the interior of the cone. In a cone covered with the oiled silk, the interior becomes so reduced in temperature that the evaporation of the ether is considerably checked.

Thinking that an inhaler would effect, amongst other advantages, considerable saving of the ether, I have had for some time my attention directed to this point, but have not as yet succeeded in suggesting any improvenient upon the one adopted by Dr. Snow.

3. The Combination of Chloroform and Ether.-My experience, in the main, confirms the opinion held by Dr. Snow, that the combination of these two liquids combines the undesirable qualities of both. When the two agents are combined in their liquid state, one never knows what one is about. I should not have considered it necessary to allude to this point, had I not seen a communication from Dr. Edis in the JOURNAL of November I6th, still advocating this method-one which, I thought, had been given up some time since. I have, however, been in the habit for some years past of administering the vapours of these two agents to the same patient with considerable advantage. I begin with chloroform ; but as soon as the pulse shows signs of failure, I commence the administration of ether, and continue its use until the pulse is restored, or as long as the patient remains quiet enough for the performance of the operation. In this manner, I believe, the cases of avoidable deaths from cardiac failure resulting from chloroform might be averted.

4. Nitrous Oxidi:- When I consider the safety, ready applicability, and the pleasantness of this agent, together with the success attending it, I confess I am surprised that surgeons do not adopt it more generally than they do for the minor operations requiring but few minutes in their performance. I have succeeded in keeping the patients under its influence for eight or ten minutes by its reapplication from time to time. I think that for some cases of operations about the eye its use would be invaluable, seeing that it produces no sickness.

\section{NOTE ON THE ADMINISTRATION OF ETHER.} By JOHN COUPER, F.R.C.S.,

Surgeon to and co-Lecturer on Surgery at the Lond nn Hospital ; Senior AssistantSurgeon to the Royal London Ophthalmic Hospital, Moorfields.

SOME years since, ether was repeatedly administered at the London Hospital, with unsatisfactory results. Although it was given in a full dose, complete narcotism was not speedily obtained, and was preceded by excitement and violent agitation of the limbs. The ether then used had not been specially prepared, and in all probability was not anhydrous. Certainly, its effects on these occasions were entirely different from those with which I have since become familiar, when pure anhydrous ether has been employed.

Having repeatedly seen the administration of ether by Dr. Joy Jeffries last August, I have since employed it exclusively, and propose now to state, briefly, the results of this short trial. The chief operations performed have been ovariotomy, lumbar colotomy, amputation through the knee-joint, partial resection of ankle, excision of tumour over the parotid gland, besides many eye operations.

The operations on the eye, for which an anæsthetic is required, demand, with few exceptions, narcotism as profound as can be safely produced. The reflex movements, produced by contact of instruments with the cornea, must cease before the operation begins; and, as a rule, they do not cease until a stage of narcotism, beyond that of insensibility to pain, is reached. I do not now inquire how far this need of profound narcotism explains the fact of a great chloroform mortality in ophthalmic practice. In regard to this point, it should be borne in mind that the number of cases in an eye-hospital requiring an anæsthetic is immensely greater than in a general hospital of equal size, and that a large apparent mortality in the former thus becomes a small death-rate. 